\title{
BMJ Open Diagnostic and prognostic accuracy of miR-21 in renal cell carcinoma: a systematic review protocol
}

\author{
Arezoo Rasti, ${ }^{1}$ Mitra Mehrazma, ${ }^{1,2}$ Zahra Madjd, ${ }^{1,2,3}$ Abbas Ali Keshtkar, ${ }^{4}$ \\ Raheleh Roudi, ${ }^{1}$ Sadegh Babashah ${ }^{5}$
}

To cite: Rasti A,

Mehrazma M, Madjd Z, et al. Diagnostic and prognostic accuracy of miR-21 in renal cell carcinoma: a systematic review protocol. BMJ Open 2016;6:e009667.

doi:10.1136/bmjopen-2015009667

- Prepublication history for this paper is available online. To view these files please visit the journal online (http://dx.doi.org/10.1136/ bmjopen-2015-009667).

Received 7 August 2015 Accepted 26 November 2015

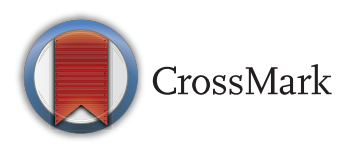

For numbered affiliations see end of article.

Correspondence to Dr Mitra Mehrazma; mitmehr@yahoo.com or

Dr Zahra Madjd; zahra.madjd@yahoo.com

\section{ABSTRACT}

Introduction: Renal cell carcinoma (RCC) is the most common neoplasm in adult kidneys. One of the most important unmet medical needs in RCC is a prognostic biomarker to enable identification of patients at high risk of relapse after nephrectomy. New biomarkers can help improve diagnosis and hence the management of patients with renal cancer. Thus, this systematic review aims to clarify the prognostic and diagnostic accuracy of miR-21 in patients with RCC.

Methods and analysis: We will include observational studies evaluating the diagnostic and prognostic roles of miR-21 in patients with renal cancer. The index test and reference standards should ideally be performed on all patients. We will search PubMed, SCOPUS and ISI Web of Science with no restriction of language. The outcome will be survival measures in adult patients with RCC. Study selection and data extraction will be performed by two independent reviewers. QUADAS-1 will be used to assess study quality. Publication bias and data synthesis will be assessed by funnel plots and Begg's and Egger's tests using Stata software V.11.1.

Ethics and dissemination: No ethical issues are predicted. These findings will be published in a peerreviewed journal and presented at national and international conferences.

Trail registration number: This systematic review protocol is registered in the PROSPERO International Prospective Register of Systematic Reviews, registration number CRD42015025001.

\section{INTRODUCTION}

Renal cell carcinoma (RCC) is the most common neoplasm in adult kidneys. ${ }^{1}$ The incidence of RCC is increasing by approximately $20 \%$ per annum. ${ }^{2}$ Distinguishing the RCC subtypes is of clinical importance because they have different prognoses and subsequently require different management plans. ${ }^{3}$ The most common form of RCC is the clear subtype RCC (ccRCC) that accounts for $75 \%-80 \%$ of cases; other less common subtypes include papillary RCC

\section{Strengths and limitations of this study}

- This systematic review, for the first time, will evaluate the prognostic and diagnostic accuracy of miR-21 in patients with renal cell carcinoma through a search of several databases without placing restrictions on language.

- The study screening, data extraction and assessment of risk of bias of the current study will be conducted by two researchers independently.

- We expect some potential heterogeneities between previous studies, including stage and histological grade in patient samples.

(pRCG), chromophobe RCG (chRCG) and collecting duct carcinoma. ${ }^{4}$ Although surgery is curative for the localised disease, a significant proportion of patients relapse or metastasise. Metastatic RCC is difficult to treat. The 5-year survival rate for metastatic RCC is dismal at less than $10 \% .^{5}$ At present, there is an absence of biomarkers for the early detection and follow-up of the disease, which is responsible for the late diagnosis and the subsequent poor prognosis. ${ }^{6}$

Factors influencing RCC prognosis can be classified into anatomic, histological, clinical and molecular. ${ }^{2}$ Pathological stage, based on the size and extent of invasion by the tumour, is the most accurate indicator of prognosis. ${ }^{7}$ Recent evidence shows that molecular signatures can classify RCC subtypes more accurately than morphological characteristics. $^{8-10}$ Micro RNAs (miRNAs) are small non-coding RNA nucleotides that post-transcriptionally regulate the expression of their target proteins. ${ }^{4}$ The first report suggesting a role of miRNAs in cancer was published in 2002. ${ }^{11}$

miRNAs are actively involved in kidney cancer pathogenesis. ${ }^{12} 13$ Recently, the clinical implications of miRNAs in RCC have been systematically reviewed; ${ }^{14}$ moreover, the potential roles of miRNAs as prognostic and 
diagnostic tools in RCC as well as its subtypes have been reported in several studies. ${ }^{4} 15-34$

Among the numerous miRNAs, miR-21 has received specific attention because of its relationship with multiple cancers. It is upregulated in several tumours, including cancers of the breast, lung, colon, pancreas, prostate and stomach. ${ }^{35-38}$ The effect of miR-21 on carcinogenesis and tumour progression has been experimentally analysed. ${ }^{39}$ miR-21 regulates epithelialmesenchymal transition phenotype and hypoxiainducible factor- $1 \alpha$ expression in breast cancer stem cell-like cells and contributes to cell migration in metastasis. ${ }^{40}$ The global prediction role of miR-21 for survival in patients with various carcinomas has been summarised in a meta-analysis. ${ }^{41}$

To date, several researchers have published their data on the prognostic value of miR-21 in RCC and raised concerns about its prognostic accuracy as a biomarker; however, these issues remain questionable. 4171931 This systematic review aims to evaluate the diagnostic and prognostic accuracy of miR-21 in patients with RCC.

\section{OBJECTIVES}

The primary objectives are to determine the diagnostic and prognostic accuracy of miR-21 in patients with RCC. The secondary objectives include determining the diagnostic and prognostic accuracy of miR-21 in RCG subtypes together with the evaluation of heterogeneity and its potential sources in primary studies.

\section{METHODS}

The methods adopted for this systematic review have been developed in accordance with the guidelines detailed on the PRISMA checklist. In addition, the PRISMA Flow Diagram will be employed to describe the flow of information through the different phases of this systematic review. ${ }^{42}$ The protocol of this systematic review has been registered in PROSPERO 2015 (registration number: CRD42015025001). The Preferred Reporting Items for Systematic reviews and Meta-Analyses for Protocols 2015 (PRISMA-P 2015) have been used for preparing and reporting the protocol of this systematic review. ${ }^{43}$

\section{INCLUSION CRITERIA}

\section{Types of studies}

Observational studies (cross-sectional, case-control and cohort) will be included. Studies assessing animal models, review articles, proceedings, conferences and case series on patients dead from RCC will be excluded.

\section{Participants}

Adult patients of both sexes with a diagnosis of RCC will be included. Tumour classification and stage will be diagnosed according to the TNM system and 2004 WHO classification. ${ }^{44} 45$
Index test

miR-21

miRNAs are highly conserved small non-coding RNA strands, approximately 22 nucleotides in length, that post-transcriptionally regulate gene expression and appear to be modulators of urological cancers. ${ }^{46} \quad 47$ miR-21 is one of the most frequently studied cancerrelated miRNAs and is dysregulated in most cancers by acting as an oncogene. ${ }^{48}$

RCC considerably exploits the upregulation of miR-21 in comparison with healthy kidneys. ${ }^{49}$ A significant difference exists between the expression levels of miR-21and RCC subtypes with respect to the highest levels in ccRCC and pRCC subtypes. miR-21 levels are associated with higher stage and grade, and hence its expression level can be used as a diagnostic marker in distinguishing RCC subtypes. ${ }^{4}$ The expression level of miR-21 is correlated with a span of 5-year survival and pathological stage in RCC. ${ }^{49}$ Patients who are miR-21 positive have statistically shorter disease-free survival (DFS) and overall survival (OS) rates. ${ }^{4}$ In the current review, miR-21 detection in RCC tissues will be defined on the basis of quantitative real-time PCR (qRT-PCR).

There are several steps for miR-21 detection in RCC tissues based on qRT-PCR, which are as follows:

1. Total RNA is extracted from RCC samples (formalinfixed, paraffin-embedded tissues or serum) using commercial kits, according to the manufacturer's protocol.

2. RNA concentrations and A260/280 ratio with a Nano Drop are determined.

3. The resulting miRNA for qRT-PCR is retained, and miRNA expression is measured by TaqMan miRNA assays.

4. Reverse transcription of miR-21 with a specified amount of total RNA using the TaqMan MicroRNA-specific Reverse Transcription Kit is performed.

5. The cycling conditions for miR-21 are selected according to the manufacturer's protocols.

6. All reactions are performed in triplicates.

7. The values of miR-21 with respect to a small nuclear RNA as a reference gene are normalised.

8. The relative overexpression or underexpression of miR-21 in patient samples is compared to that in normal subjects by the $\Delta \Delta \mathrm{Ct}$ method. ${ }^{4} 1931$

\section{Gold standards}

Studies will be eligible for this review if the clinical diagnostic criteria recommended by the WHO classification and TNM system of the renal tumours of adults are used as primary reference standards. The pathological diagnosis of RCC is recommended for all renal masses existent in radical nephrectomy or biopsies. Since clinical practice guidelines are routinely updated, we will use the criteria of reference standard that the primary studies adopted. Survival measures such as DFS, OS, recurrence- 
free survival (RFS) and HR with 95\% CI will be used as reference standards for prognostic accuracy.

\section{Target conditions}

The target conditions for diagnostic and prognostic values will be verified by RCC diagnosis and survival measures, respectively.

\section{Outcomes}

The primary outcomes will be all survival measures of patients with RCC. The secondary outcomes will include the determination of prognostic accuracy of miR-21 in different subtypes of renal cancer, as well as the determination of heterogeneity and its potential sources.

\section{SEARCH METHODS FOR IDENTIFICATION OF STUDIES Electronic searches}

We will search the following databases: PubMed, Web of Science and Scopus, till 31 July 2015.

\section{PubMed search strategy}

MeSH tags were found in PubMed. The details of the PubMed database search strategy and syntax are sequentially provided below.

1. miR21

2. miR-21

3. microRNA-21

4. miRNA-21

5. hsa-mir-21

6. 1 or 2 or 3 or 4 or 5

7. Renal

8. Kidney

9. 7 or 8

10. 6 and 9

[miR21 (tiab) OR microRNA-21(tiab) OR miRNA-21 (tiab) OR hsa-mir-21(tiab) OR miR-21(tiab)] AND [kidney (tiab) OR renal (tiab)].

\section{Other resources}

Reference lists of relevant primary studies, reviews and key journals will be searched for additional studies.

\section{Data collection and analysis}

A data extraction form will be developed, and study data will be independently assessed and extracted by two reviewers, AR and RR.

The following data will be extracted from all the included studies:

1. Study characteristics (author, year of publication, study design, setting, locations and patient enrolment strategies).

2. Test characteristics (test type, test condition, prespecified cut-off values, sampling protocol and criteria for test positivity).

3. Reference standards (histopathological diagnosis or criteria of the guideline used).
4. Participants' characteristics (age, gender, ethnicity, disease type, tumour sizes and tumour stages).

5. Statistics for meta-analysis, including true positives, false positives, true negatives, false negatives, sensitivity, specificity, positive and negative likelihood ratios, area under the curve (AUC) for diagnostic value, OS, DFS and RFS for prognostic values.

Unavailable information from included studies will be sought from authors by email. Any discrepancies will be resolved by consensus between AR and RR.

\section{Assessment of methodological quality}

The methodological quality of primary studies will be assessed by a revised tool devised for QUADAS-1 quality assessment. ${ }^{50}$ The defined questions will be answered as yes, no or unclear, and the score of each article will be calculated. Two authors (AR and RR) will initially complete the pilot QUADAS-1 items in three studies. If the authors do not agree, we will refine the tool, and then the updated tool will be applied to complete the assessment for all included studies. Disagreements will be resolved by consensus.

\section{Assessment of heterogeneity}

To investigate heterogeneity, we will include the study design (prospective or retrospective and year of publication), population characteristics (gender, ethnicity, age, disease types and stage distribution), test characteristics (cut-off value, test type and the total number of tests per screening round) and versions of reference standards as our study-level variables. We will test these study-level covariates in the bivariate model in the common threshold or add them to the Rutter and Gatsonis HSROC model to evaluate the heterogeneity in the test threshold, diagnostic accuracy and the shape of curves. The likelihood ratio test will be used to determine the statistical significance of the covariates included in the models. $^{51}$

\section{Assessment of reporting bias}

The publication bias will be assessed by funnel plots (ie, plots of study results against precision) and Begg's and Egger's tests.

\section{Statistical analysis and data synthesis \\ Descriptive analysis}

All included studies will be overviewed and presented in two separate tables. The first table will provide details on the study quality according to the review-specific QUADAS-1 tool. The other table will include the study design, participants, test characteristics, results, outcomes and reference standards. The following test characteristics will be extracted into $2 \times 2$ tables (TP, FP, $\mathrm{TN}$ and FN) for all included studies. Study-specific estimations of sensitivity and specificity with $95 \%$ CI will be displayed in forest plots using Review Manager (V.5.2). These graphical displays will reveal the variations in accuracy among the studies and the different types and 
brands of the index test. The collected data about the outcomes such as DFS, OS, RFS and HR with 95\% CI will be used for calculating the prognostic value of the index test.

\section{Inferential statistics}

All the included studies will be synthesised after a systemic review. Study-specific HR, sensitivity and specificity estimates will be pooled using a fixed effect model, if no significant heterogeneity exists; otherwise, a random effect model will be applied. The extent of heterogeneity throughout the studies will be checked using the $\chi^{2}$ test and $\mathrm{I}^{2}$ test; $\mathrm{p}=0.10$ and/or $\mathrm{I}^{2}>50 \%$, indicating significant heterogeneity. To evaluate the sources of heterogeneity, subgroup analysis and meta-regression will be performed.

The potential for publication bias will be assessed using funnel plots, Begg's rank correlation method and Egger's weighted regression method. A $p$ value of $\leq 0.05$ will be considered to be statistically significant.

All the mentioned analyses will be performed using STATA V.11.1 software (StataCorp LP, College Station, Texas, USA).

\section{Sensitivity analysis}

A sensitivity analysis will be conducted to test the impact of the results with respect to the methodological quality items rated by the QUADAS-1 tool criteria. We will also implement sensitivity analyses to explore the impacts of methodological quality and sample size on the robustness of review conclusions. Meta-analyses will be repeated after excluding studies with lower methodological quality and studies with sample sizes much larger than those of other studies. Sensitivity analyses will be reported with a summary table, and reviewed conclusions will be interpreted by making comparisons between the two meta-analyses.

\section{Subgroup analyses}

Subgroup analyses will be conducted according to the region, gender, quality, sample size and cancer subtype.

\section{DISCUSSION}

Despite the comprehensive understanding of the biology of RCC and recent advances in novel therapeutic strategies, the lack of molecular biomarkers for the early detection and classification of RCC and the poor prognosis for these patients are major complications. miR-21 is a tumour marker that is upregulated in RCC and in several tumours. In RCC, miR-21 is involved in cellular mechanisms such as growth, apoptosis, cell cycle, invasion and migration of tumour cells.

Our systematic review will clarify the prognostic and diagnostic accuracy of miR-21 in patients with RCC, which would allow patients, clinicians and researchers to determine the diagnostic performance of miR-21 for the detection of RCC, particularly early stage RCC, and the potential role of this marker in the prognosis of patients with RCC. This systematic review will also help policymakers and guideline developers in the management of patients with RCC.

Author affiliations

${ }^{1}$ Oncopathology Research Center, Iran University of Medical Sciences, Tehran, Iran

${ }^{2}$ Department of Pathology, Iran University of Medical Sciences, Tehran, Iran ${ }^{3}$ Department of Molecular Medicine, Faculty of Advanced Technologies in Medicine, Iran University of Medical Sciences, Tehran, Iran

${ }^{4}$ Department of Health Sciences Education Development, School of Public Health, Tehran University of Medical Sciences, Tehran, Iran

${ }^{5}$ Department of Molecular Genetics, Faculty of Biological Sciences, Tarbiat Modares University, Tehran, Iran

Acknowledgements The authors would like to acknowledge the efforts of $\mathrm{Mr}$ Rahul Krishan Arora and Mr Alireza Ahmadi Raad for their diligent and thoughtful editing of the manuscript.

Contributors MM, ZM, AR and AAK contributed to the concept and study design. AAK and AR developed the search strategies and contributed to the implementation and analysis of the studies. AR contributed to the initial drafting and critical revision and approved the manuscript for submission. RR and $S B$ revised the manuscript. AR and RR will also screen potential studies, extract data and assess their quality. Any discrepancies will be resolved by consensus between the two authors.

\section{Competing interests None declared}

Provenance and peer review Not commissioned; externally peer reviewed.

Data sharing statement All recorded data from the data extraction process will be available on request to the extent that they are not included in the systematic review article.

Open Access This is an Open Access article distributed in accordance with the Creative Commons Attribution Non Commercial (CC BY-NC 4.0) license, which permits others to distribute, remix, adapt, build upon this work noncommercially, and license their derivative works on different terms, provided the original work is properly cited and the use is non-commercial. See: http:// creativecommons.org/licenses/by-nc/4.0/

\section{REFERENCES}

1. Bukowski RM. Prognostic factors for survival in metastatic renal cell carcinoma:update 2008. Cancer 2009;115:2273-81.

2. Ljungberg B, Cowan NC, Hanbury DC, et al. EAU guidelines on renal cell carcinoma: the 2010 update. Eur Urol 2010;58:398-406.

3. Youssef YM, White NM, Grigull J, et al. Accurate molecular classification of kidney cancer subtypes using microRNA signature. Eur Urol 2011:59:721-30.

4. Faragalla $\mathrm{H}$, Youssef YM, Scorilas $\mathrm{A}$, et al. The clinical utility of miR-21 as a diagnostic and prognostic marker for renal cell carcinoma. J Mol Diagn 2012;14:385-92.

5. Motzer RJ, Bander NH, Nanus DM. Renal-cell carcinoma. N Engl J Med 1996;335:865-75.

6. SlabyO Jancovicova J, Lakomy R, et al. Expression of miRnA- 106b in conventional renal cell carcinoma is a potential marker for prediction of early metastasis after nephrectomy. J Exp Clin Cancer Res 2010;29:90.

7. Hanahan D, Weinberg RA. The hallmarks of cancer. Cell 2000;100:57-70.

8. White NM, Bao TT, Grigull J, et al. miRNA profiling for clear cell renal cell carcinoma: biomarker discovery and identification of potential controls and consequences of miRNA dysregulation. $J$ Urol 2011;186:1077-83.

9. White NM, Yousef GM. MicroRNAs: exploring a new dimension in the pathogenesis of kidney cancer. BMC Med 2010;8:65.

10. White NM, Yousef GM. Translating molecular signatures of renal cell carcinoma into clinical practice. J Urol 2011;186:9-11.

11. Eiring AM, Harb JG, Neviani $P$, et al. miR-328 functions as an RNA decoy to modulate hnRNP E2 regulation of mRNA translation in leukemic blasts. Cell 2010;140:652-65. 
12. Chow TF, Mankaruos M, Scorilas A, et al. The miR-17-92 cluster is over expressed in and has an oncogenic effect on renal cell carcinoma. J Urol 2010;183:743-51.

13. White NM, Bui A, Mejia-Guerrero S, et al. Dysregulation of kallikrein-related peptidases in renal cell carcinoma: potential targets of miRNAs. Biol Chem 2010;391:411-23.

14. Li M, Wang $\mathrm{Y}$, Song $\mathrm{Y}$, et al. MicroRNAs in renal cell carcinoma: a systematic review of clinical implications (Review). Oncol Rep 2015;33:1571-8.

15. Wang $\mathrm{C}, \mathrm{Hu} \mathrm{J}, \mathrm{Lu} \mathrm{M}$, et al. A panel of five serum miRNAs as a potential diagnostic tool for early-stage renal cell carcinoma. Sci Rep 2015;5:7610.

16. Fu Q, Liu Z, Pan D, et al. Tumor miR-125b predicts recurrence and survival of patients with clear-cell renal cell carcinoma after surgical resection. Cancer Sci 2014;105:1427-34.

17. Chen X, Ruan A, Wang X, et al. miR-129-3p, as a diagnostic and prognostic biomarker for renal cell carcinoma, attenuates cell migration and invasion via downregulating multiple metastasis-related genes. J Cancer Res Clin Oncol 2014;140:1295-304.

18. Fritz HK, Lindgren $\mathrm{D}$, Ljungberg $\mathrm{B}$, et al. The miR $21 / 10 \mathrm{~b}$ ratio as a prognostic marker in clear cell renal cell carcinoma. Eur J Cancer 2014;50:1758-65.

19. Wang J, Zhao J, Shi M, et al. Elevated expression of miR-210 predicts poor survival of cancer patients: a systematic review and meta-analysis. PLOS ONE 2014;9:e89223.

20. Vergho D, Kneitz S, Rosenwald A, et al. Combination of expression levels of miR-21 and miR-126 is associated with cancer-specific survival in clear-cell renal cell carcinoma. BMC Cancer 2014;14:25.

21. Teixeira AL, Ferreira M, Silva J, et al. Higher circulating expression levels of miR-221 associated with poor overall survival in renal cell carcinoma patients. Tumor Biol 2014;35:4057-66.

22. Gowrishankar B, Ibragimova I, Zhou Y, et al. MicroRNA expression signatures of stage, grade, and progression in clear cell RCC. Cancer Biol Ther 2014;15:329-41.

23. Heinzelmann J, Unrein A, Wickmann U, et al. MicroRNAs with prognostic potential for metastasis in clear cell renal cell carcinoma: a comparison of primary tumors and distant metastases. Ann Surg Oncol 2014;21:1046-54.

24. Silva-Santos R, Costa-Pinheiro P, Luis A, et al. MicroRNA profile: a promising ancillary tool for accurate renal cell tumour diagnosis. $\mathrm{Br} \mathrm{J}$ Cancer 2013;109:2646-53

25. Li H, Zhao J, Zhang J, et al. MicroRNA-217, down-regulated in clear cell renal cell carcinoma and associated with lower survival, suppresses cell proliferation and migration. Neoplasma 2012;60:511-15.

26. Wang G, Chen L, Meng J, et al. Overexpression of microRNA-100 predicts an unfavorable prognosis in renal cell carcinoma. Int Urol Nephrol 2013:45:373-9.

27. Si T, Liu C, Xu K, et al. [Association of miR-199a expression with clinicopathologic characteristics and prognosis of renal cell carcinoma]. Nan Fang Yi Ke Da Xue Xue Bao 2012;32:1568-71.

28. Wu X, Weng L, Li X, et al. Identification of a 4-microRNA signature for clear cell renal cell carcinoma metastasis and prognosis. PloS ONE 2012;7:e35661.

29. Hauser $S$, Wulfken LM, Holdenrieder $S$, et al. Analysis of serum microRNAs (miR-26a-2*, miR-191, miR-337-3p and miR-378) as potential biomarkers in renal cell carcinoma. Cancer Epidemiol 2012;36:391-4

30. Zhao X, Zhao Z, Xu W, et al. Down-regulation of miR-497 is associated with poor prognosis in renal cancer. Int J Clin Exp Pathol 2015;8:758.

31. Khella HW, Scorilas A, Mozes R, et al. Low expression of miR-126 is a prognostic marker for metastatic clear cell renal cell carcinoma. Am J Pathol 2015;185:693-703.
32. Vergho DC, Kneitz S, Kalogirou C, et al. Impact of miR-21, miR-126 and miR-221 as prognostic factors of clear cell renal cell carcinoma with tumor thrombus of the inferior vena cava. PLoS One 2014;9: e109877.

33. Zhao J-J, Chen P-J, Duan R-Q, et al. Up-regulation of miR-630 in clear cell renal cell carcinoma is associated with lower overal survival. Int J Clin Exp Pathol 2014;7:3318.

34. Goto K, Oue N, Shinmei S, et al. Expression of miR-486 is a potential prognostic factor after nephrectomy in advanced renal cell carcinoma. Mol Clin Oncol 2013;1:235-40.

35. Markou A, Tsaroucha EG, Kaklamanis L, et al. Prognostic value of mature microRNA-21 and microRNA-205 overexpression in nonsmall cell lung cancer by quantitative real-time RT-PCR. Clin Chem 2008;54:1696-704.

36. Slaby O, Svoboda M, Fabian P, et al. Altered expression of miR-21, miR-31, miR-143 and miR-145 is related to clinicopathologic features of colorectal cancer. Oncology 2007;72:397-402.

37. Yan L-X, Huang X-F, Shao Q, et al. MicroRNA miR-21 overexpression in human breast cancer is associated with advanced clinical stage, lymph node metastasis and patient poor prognosis. RNA 2008;14:2348-60.

38. Hui $\mathrm{A}$, How $\mathrm{C}$, Ito $\mathrm{E}$, et al. Micro-RNAs as diagnostic or prognostic markers in human epithelial malignancies. BMC Cancer 2011;11:500.

39. White NM, Fatoohi E, Metias M, et al. Metastamirs: a stepping stone towards improved cancer management. Nat Rev Clin Oncol 2011;8:75-84

40. Han M, Wang Y, Liu M, et al. MiR-21 regulates epithelialmesenchymal transition phenotype and hypoxia-inducible factor- $1 \alpha$ expression in third-sphere forming breast cancer stem cell-like cells. Cancer Sci 2012;103:1058-64.

41. Fu X, Han $\mathrm{Y}, \mathrm{Wu} \mathrm{Y}$, et al. Prognostic role of microRNA-21 in various carcinomas: a systematic review and meta-analysis. Eur J Clin Invest 2011:41:1245-53.

42. Liberati A, Altman DG, Tetzlaff $\mathrm{J}$, et al. The PRISMA statement for reporting systematic reviews and meta-analyses of studies that evaluate health care interventions: explanation and elaboration. Ann Intern Med 2009;151:W-65-94.

43. Moher D, Shamseer L, Clarke M, et al. Preferred reporting items for systematic review and meta-analysis protocols (PRISMA-P) 2015 statement. Syst Rev 2015;4:1.

44. Lopez-Beltran A, Scarpelli M, Montironi R, et al. 2004 WHO classification of the renal tumors of the adults. Eur Urol 2006;49:798-805

45. Srigley JR, Amin MB, Delahunt B, et al. Protocol for the examination of specimens from patients with invasive carcinoma of renal tubular origin. Arch Pathol Lab Med 2010;134:e25-30.

46. Catto JW, Alcaraz A, Bjartell AS, et al. MicroRNA in prostate, bladder, and kidney cancer: a systematic review. Eur Urol 2011;59:671-81.

47. Bartel DP. MicroRNAs: genomics, biogenesis, mechanism, and function. Cell 2004;116:281-97.

48. Kumarswamy R, Volkmann I, Thum T. Regulation and function of miRNA-21 in health and disease. RNA Biol 2011;8:706-13.

49. Zaman MS, Shahryari V, Deng G, et al. Up-regulation of microRNA-21 correlates with lower kidney cancer survival. PLOS ONE 2012;7:e31060.

50. Whiting P, Rutjes AW, Reitsma JB, et al. The development of QUADAS: a tool for the quality assessment of studies of diagnostic accuracy included in systematic reviews. BMC Med Res Methodol 2003;3:25.

51. Rutter CM, Gatsonis CA. A hierarchical regression approach to meta-analysis of diagnostic test accuracy evaluations. Stat Med 2001;20:2865-84. 